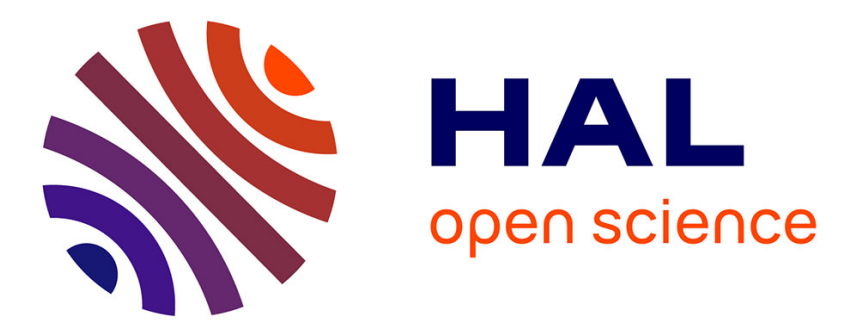

\title{
Mesure de la fonction de distribution des vitesses d'un jet moléculaire
}

\author{
J.P. Berthier, A. Constans, G. Daury, P. Lostis
}

\section{To cite this version:}

J.P. Berthier, A. Constans, G. Daury, P. Lostis. Mesure de la fonction de distribution des vitesses d'un jet moléculaire. Revue de Physique Appliquée, 1971, 6 (4), pp.433-439. 10.1051/rphysap:0197100604043300 . jpa-00243567

\section{HAL Id: jpa-00243567 https://hal.science/jpa-00243567}

Submitted on 1 Jan 1971

HAL is a multi-disciplinary open access archive for the deposit and dissemination of scientific research documents, whether they are published or not. The documents may come from teaching and research institutions in France or abroad, or from public or private research centers.
L'archive ouverte pluridisciplinaire HAL, est destinée au dépôt et à la diffusion de documents scientifiques de niveau recherche, publiés ou non, émanant des établissements d'enseignement et de recherche français ou étrangers, des laboratoires publics ou privés. 


\title{
MESURE DE LA FONCTION DE DISTRIBUTION DES VITESSES D'UN JET MOLÉCULAIRE
}

\author{
J. P. BERTHIER, A. CONSTANS, G. DAURY et P. LOSTIS \\ Institut d'Optique, 3, boulevard Pasteur, Paris $15^{\mathrm{e}}$
}

(Reçu le 1er juin 1971, révisé le 30 juin 1971)

\begin{abstract}
Résumé. - La mesure des distributions de vitesse des molécules d'un jet moléculaire est habituellement réalisée au moyen de la méthode de Fizeau. Cette méthode donne directement une densité de probabilité. Les auteurs développent ici une nouvelle méthode fournissant une fonction de répartition ; la mesure opérée sur une grandeur intégrale permet d'obtenir des résultats précis à partir d'une grandeur entachée de bruit. On passe alors à la densité de probabilité en opérant une déconvolution numérique.

Abstract. - The measurement of a molecular beam velocity distribution is usually performed by the Fizeau method, which directly provides a probability density function.

This paper presents an original method for the distribution function measurement ; the measurement of this function is made from an integral quantity and allows to get precise results from a noisy environment quantity. Then we proceed to the probability density function through numerical deconvolution.
\end{abstract}

I. Introduction. - Dans les études utilisant des jets moléculaires, la connaissance du temps de vol des molécules du jet et de la fonction de distribution des vitesses de ces molécules est essentielle.

Pour effectuer la mesure de ces paramètres, on se heurte la plupart du temps à deux problèmes :

- Les méthodes de mesure utilisées conduisent à des résultats très imprécis à cause de l'impossibilité technologique dans la mise en œuvre de ces méthodes. En effet, ces méthodes supposent qu'il est possible de découper un jet moléculaire en paquets infiniment minces.

- L'information recueillie est noyée dans un bruit de fond très important.

Nous avons donc cherché à développer une méthode de mesure tenant compte des impératifs technologiques, et à réaliser un appareillage permettant de lutter efficacement contre le bruit. Nous avons ainsi pu obtenir des résultats avec une précision de l'ordre de $5 \%$.

L'appareil que nous décrivons dans cet article a été particulièrement orienté pour des mesures dans un jet moléculaire de particules neutres à faible vitesse $(<2000 \mathrm{~m} / \mathrm{s})$.

II. Principe. - Supposons d'abord que nous puissions établir à un instant donné $t_{0}$ et pendant un temps extrêmement bref un jet moléculaire issu d'une source S. La "bouffée » de matière ainsi produite contient des molécules dont les vitesses sont distribuées suivant une certaine loi, mais l'ensemble se propage avec une vitesse $U$, qui est la vitesse moyenne des $n$ molécules du paquet :

$$
U=\frac{1}{n} \sum_{i=1}^{n} v_{\mathrm{i}}
$$

$\mathrm{Au}$ fur et à mesure de la propagation, il s'opère un tri en vitesse ; le paquet de matière, qui initialement était très serré, s’étale (Fig. 1).

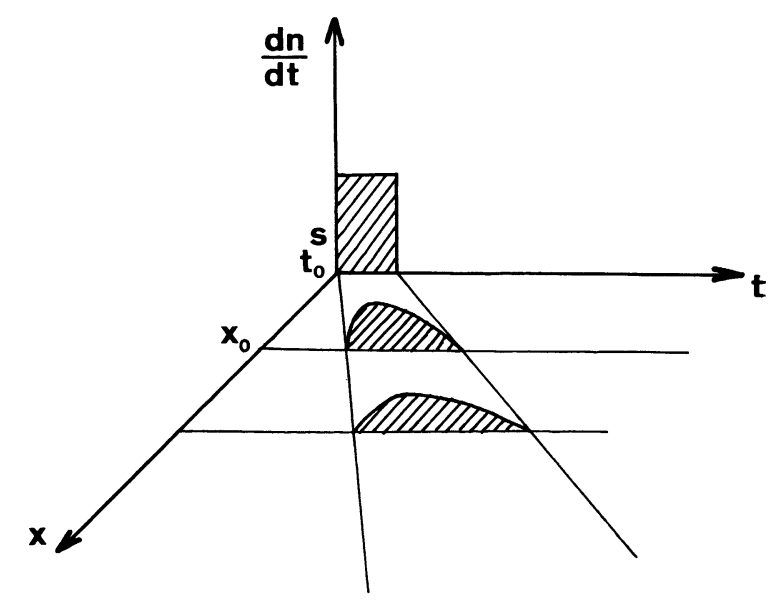

FIG. 1.

Si on mesure le nombre $(\mathrm{d} n(t) / \mathrm{d} t)_{x_{0}}$ de molécules qui passent à chaque instant à travers un plan perpendiculaire au jet et situé à une distance $x_{0}$ de la source, 
on obtient une courbe ayant l'allure de la figure 2 : les molécules, qui sont parties ensemble de la source $S$, arrivent au plan $x=x_{0}$ à des instants différents, et la courbe obtenue représente la fonction de répartition en temps de vol des molécules du jet. On en déduit facilement la répartition en vitesse, puisque $x_{0}$ est connu.

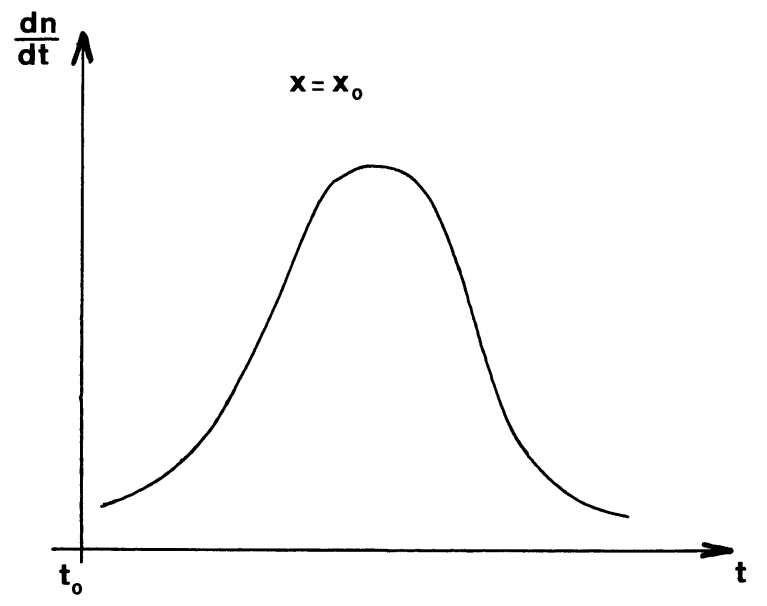

FIG. 2.

Cette méthode de tri très simple n'est cependant pas réalisable parfaitement. Il est en effet impossible d'obtenir un paquet de matière émis brutalement pendant un temps infiniment bref et contenant un nombre de molécules suffisant pour représenter une population statistique. En réalité, le débit $(\mathrm{d} n / \mathrm{d} t)_{s}$ de molécules émises par la source $\mathrm{S}$, en fonction du temps à l'allure de la figure 2 .

Cherchons donc à interpréter les courbes obtenues dans le cas où le paquet de matière est émis par la source pendant un temps non nul.

Supposons que nous connaissions simultanément la courbe $(\mathrm{d} n / \mathrm{d} t)_{s}$ correspondant au débit de la source (plan $x=0)$ et celle $(\mathrm{d} n(t) / \mathrm{d} t)_{x_{0}}$ correspondant au nombre de molécules qui traversent le plan $x=x_{0}$ à chaque instant (Fig. 3).

La courbe $(\mathrm{d} n / \mathrm{d} t)_{\text {s }}$ peut être décomposée en $n$ petits secteurs de largeur $\Delta t_{\mathrm{i}}$ très minces. On peut alors

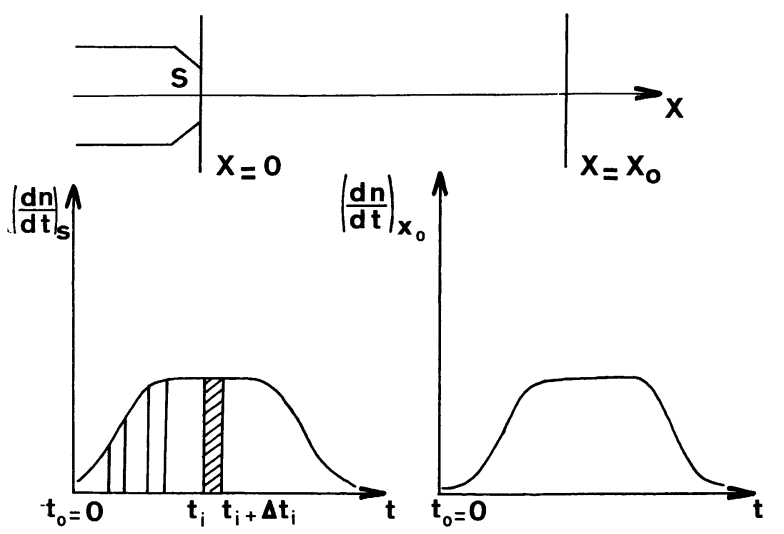

FIG. 3. considérer la courbe $(\mathrm{d} n(t) / \mathrm{d} t)_{x_{0}}$ comme résultant de la superposition de $n$ courbes élémentaires, chacun de ces motifs élémentaires représentant, à un facteur constant près, la courbe de répartition en temps de vol dont nous avons parlé. Ainsi, à un petit secteur $\Delta t_{\mathrm{i}}$ de la courbe $(\mathrm{d} n / \mathrm{d} t)_{s}$ représentant le débit de la source émis de l'instant $t_{\mathrm{i}}$ à l'instant $t+\Delta t_{\mathrm{i}}$, correspond un motif élémentaire qui se développe dans le temps à partir de l'instant $t_{\mathrm{i}}$ : la courbe correspondante est donc une fonction de $t-t_{\mathrm{i}}$.

Nous appellerons $S\left(t_{\mathrm{i}}\right) \times f\left(t-t_{\mathrm{i}}\right)$ la courbe élémentaire correspondant au petit secteur commençant à l'instant $t_{\mathrm{i}}, S\left(t_{\mathrm{i}}\right)$ étant la valeur de $(\mathrm{d} n / \mathrm{d} t)_{s}$ à l'instant $t_{\mathrm{i}}$, et $f\left(t-t_{\mathrm{i}}\right)$ la fonction de distribution des temps de vol des molécules parties de la source entre l'instant $t_{\mathrm{i}}$ et $t_{\mathrm{i}}+\Delta t_{\mathrm{i}}$.

Supposons d'abord, pour simplifier, que la courbe $(\mathrm{d} n / \mathrm{d} t)_{s}$ est une fonction créneau (Fig. 4) d'amplitude $S_{0}$. La courbe $(\mathrm{d} n(t) / \mathrm{d} t)_{x_{0}}$ est la somme des motifs élémentaires résultant, soit :

$$
\left(\frac{\mathrm{d} n(t)}{\mathrm{d} t}\right)_{x_{0}}=\int_{0}^{t} S_{0} f\left(t-t_{\mathrm{i}}\right) \mathrm{d} t_{\mathrm{i}}
$$

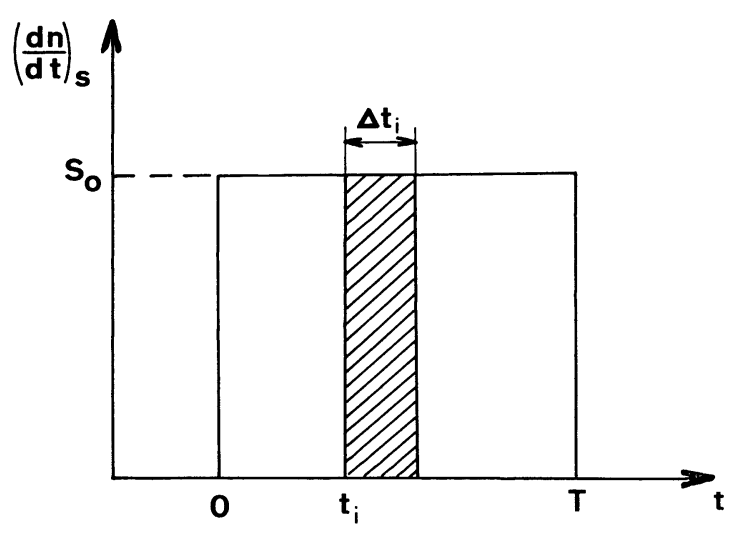

FIG. 4.

étant entendu qu'à partir de l'instant $T$ il n'y a plus de nouveau motif élémentaire, donc que si $t>T$ :

$$
\left(\frac{\mathrm{d} n(t)}{\mathrm{d} t}\right)_{x_{0}}=\int_{0}^{T} S_{0} f\left(t-t_{\mathrm{i}}\right) \mathrm{d} t_{\mathrm{i}} .
$$

Plus généralement, si la fonction source est $S\left(t_{\mathrm{i}}\right)$, on aura :

$$
\left(\frac{\mathrm{d} n(t)}{\mathrm{d} t}\right)_{x_{0}}=\int_{0}^{t} S\left(t_{\mathrm{i}}\right) f\left(t-t_{\mathrm{i}}\right) \mathrm{d} t_{\mathrm{i}} .
$$

Reprenons le cas de la formule (1) correspondant à une fonction $(\mathrm{d} n / \mathrm{d} t)_{s}$ en créneau. En dérivant par rapport à $t$, on $\mathrm{a}$ :

$$
\frac{\mathrm{d}}{\mathrm{d} t}\left[\left(\frac{\mathrm{d} n(t)}{\mathrm{d} t}\right)_{x_{0}}\right]=S_{0} \int_{0}^{t} \frac{\mathrm{d}}{\mathrm{d} t}\left[f\left(t-t_{\mathrm{i}}\right)\right] \mathrm{d} t_{\mathrm{i}} .
$$

Or :

$$
\frac{\mathrm{d}}{\mathrm{d} t}\left[f\left(t-t_{\mathrm{i}}\right)\right]=-\frac{\mathrm{d}}{\mathrm{d} t_{\mathrm{i}}}\left[f\left(t-t_{\mathrm{i}}\right)\right]
$$


d'où :

$$
\begin{aligned}
\frac{\mathrm{d}}{\mathrm{d} t}\left[\left(\frac{\mathrm{d} n(t)}{\mathrm{d} t}\right)_{x_{0}}\right]=-S_{0} \int_{0}^{t} \mathrm{~d}[f(t & \left.\left.-t_{i}\right)\right]= \\
& =S_{0}[f(t)-f(0)] .
\end{aligned}
$$

Donc :

$$
f(t)=f(0)+\frac{1}{S_{0}} \frac{\mathrm{d}}{\mathrm{d} t}\left[\left(\frac{\mathrm{d} n(t)}{\mathrm{d} t}\right)_{x_{0}}\right] \quad \text { si } \quad t \leqslant T .
$$

Dans les cas qui nous intéressent, $f(0)=0$ car il n'y a pas de molécules de vitesse infinie.

Dans le cas où $t>T$, au lieu de (3) nous aurons :

$$
\frac{\mathrm{d}}{\mathrm{d} t}\left[\left(\frac{\mathrm{d} n(t)}{\mathrm{d} t}\right)_{x_{0}}\right]=S_{0}[f(t)-f(t-T)] .
$$

Nous voyons ainsi que dans le cas où la fonction $(\mathrm{d} n / \mathrm{d} t)_{s}$ est une fonction créneau, la courbe de répartition en temps de vol est, à un facteur constant près, obtenue en prenant la pente de la courbe $(\mathrm{d} n(t) / \mathrm{d} t)_{x_{0}}$, du moins tant que $t \leqslant T$. L'analyse de la pente n'est donc exploitable que si la durée du créneau source est suffisamment longue.

Dans le cas général correspondant à la formule (2), il n'est plus possible de mener le calcul jusqu'au bout, car la fonction sous le signe somme n'est plus une fonction de $t-t_{\mathrm{i}}$. On calcule alors l'intégrale par itération. Remarquons cependant que l'allure de la courbe $(\mathrm{d} n / \mathrm{d} t)_{s}$ est dans la pratique très voisine de la figure 4. Et en adoptant comme départ de calcul l'approximation fournie par la formule (3), le calcul par itération est très rapide.

Nous voyons donc que le relevé des deux courbes $(\mathrm{d} n / \mathrm{d} t)_{s}$ et $(\mathrm{d} n(t) / \mathrm{d} t)_{x_{0}}$ nous permet de connaître la fonction de répartition en temps de vol au moyen d'un dispositif physiquement réalisable sans approximation.

Remarque. - La formule (2) obtenue peut s'écrire :

$$
\left(\frac{\mathrm{d} n(t)}{\mathrm{d} t}\right)_{x_{0}}=S(t) * f(t),
$$

* indiquant une convolution, ce qui s'exprime, d'après le théorème de Borel, par :

$$
\mathcal{N}(p)=\mathcal{S}(p) \cdot \mathcal{F}(p)
$$

en utilisant les transformées de Laplace.

L'ensemble électronique utilisé pour la mesure de $(\mathrm{d} n / \mathrm{d} t)_{x_{0}}$ fournit une tension $v(t)$. Cette tension n'est pas parfaitement proportionnelle au débit, mais on peut toujours écrire :

$$
\mathcal{V}(p)=\mathcal{A}(p) \mathcal{N}(p)
$$

$\mathcal{A}(p)$ étant l'expression en Laplace de la transmittance globale de la chaîne de mesure, d'où :

$$
\mathcal{V}(p)=\mathcal{A}(p) \cdot \mathcal{S}(p) \cdot \mathcal{F}(p)
$$

ou en posant :

$$
\begin{gathered}
\varepsilon(p)=\mathcal{A}(p) \mathcal{S}(p) . \\
\cup(p)=\varepsilon(p) \mathcal{F}(p) .
\end{gathered}
$$

Nous pouvons donc écrire en partant de la formule (2) :

$$
v(t)=K \int_{0}^{t} S^{\prime}\left(t_{\mathrm{i}}\right) f\left(t-t_{\mathrm{i}}\right) \mathrm{d} t_{\mathrm{i}}
$$

où $K$ est une constante et $S^{\prime}(t)$ une fonction tenant compte à la fois de l'allure de $(\mathrm{d} n / \mathrm{d} t)_{S}$ et de la réponse de la chaîne électronique.

III. Description de l'appareillage. - A) JET UTILISÉ, DISPOSITIF MÉCANIQUE. - Le jet étudié est produit par un générateur Kantrowitz-Grey modifié par Campargue. Son débit est très élevé (environ $10^{19}$ molécules/stéradian/s) et réglable [1], [2], [3], [4].

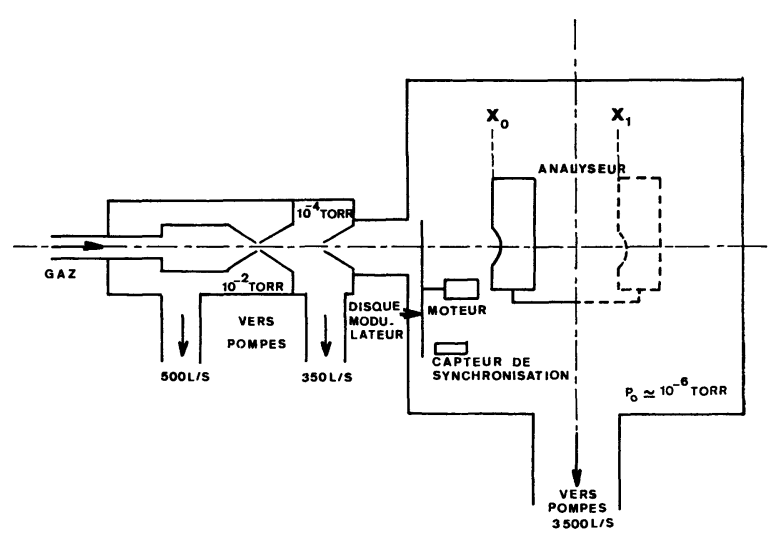

Fig. 5.

Un disque comportant des fentes et entraîné par un moteur permet de moduler le jet. Un capteur photosensible reçoit un pinceau lumineux modulé par le disque, et fournit ainsi un signal de synchronisation.

La mesure du débit du jet est faite au moyen d'une jauge d'ionisation et d'un spectromètre de masse suivi d'un multiplicateur d'électrons. Le signal obtenu est ensuite traité par un ensemble électronique décrit au paragraphe suivant.

L'analyseur ainsi formé est monté sur un ensemble mécanique permettant de le déplacer par rotation. On dispose ainsi de deux positions possibles $x_{0}$ et $x_{1}$ pour analyser le jet. La première position se trouve à environ $20 \mathrm{~cm}$ de la source, la seconde à $75 \mathrm{~cm}$.

L'ensemble comporte plusieurs chambres dans lesquelles le vide est assuré par des groupes de pompage.

B) DiSPOSITIF ÉLECTRONIQUE UTILISÉ POUR RELEVER LES COURBES: Nous avons vu que le problème de 
la mesure des temps de vol revenait à relever des courbes $(\mathrm{d} n(t) / \mathrm{d} t)_{x}$ pour $x=0$ et $x=x_{0}$, et nous avons indiqué comment, au moyen d'un analyseur de masse, nous obtenions un courant électrique proportionnel à $\mathrm{d} n(t) / \mathrm{d} t$.

Malheureusement, le signal obtenu à la sortie du détecteur est en général totalement noyé dans le bruit de fond. Ce bruit a plusieurs origines :

- un bruit d'agitation thermique des molécules du gaz résiduel (pression dans l'enceinte $=2 \times 10^{-6}$ torr) ;

- un bruit d'origine électrique provenant de la tête d'analyse ;

- un bruit dû à des parasites externes (moteurs des pompes,...) Il faut mettre en œuvre une technique très élaborée pour éliminer ce bruit.

a) Description de la chaîne de mesure. - Conformément à la figure 6 , le débit $\mathrm{d} n / \mathrm{d} t$ du jet est mesuré au moyen d'une jauge d'ionisation, suivie d'un spectromètre de masse et d'un multiplicateur d'électrons (1). L'ensemble fournit un courant proportionnel à ce débit. Le signal est préamplifié dans (2). Le jet est découpé en paquets successifs identiques au moyen du disque modulateur; le signal obtenu est un signal périodique se développant à la fréquence $f_{0}$, noyé dans du bruit. On opère en (3) un filtrage linéaire en réalisant un filtre de Fourier ; nous avons éliminé la composante continue du signal qui ne sert à rien puisque nous ne nous intéressons qu'aux flancs de montée.

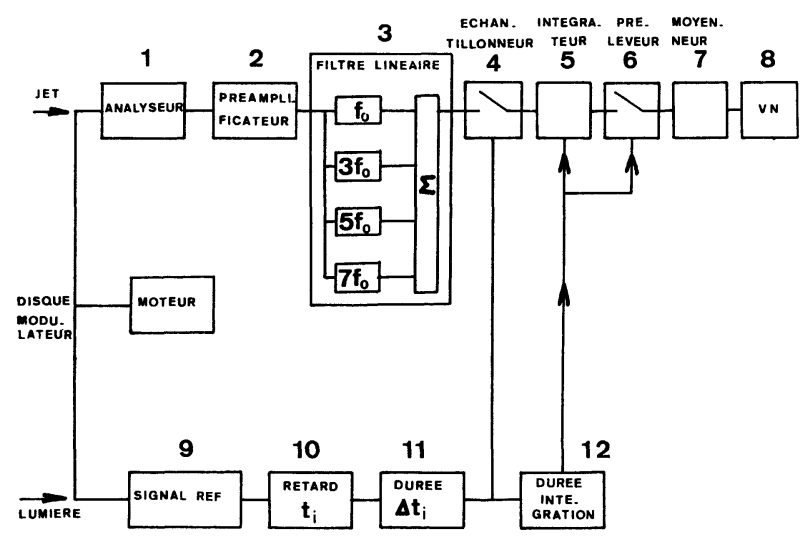

FIG. 6.

De plus, le signal que nous avons à traiter ne comporte pas d'harmoniques pairs. A la sortie du filtre linéaire, un interrupteur (4) permet d'échantillonner le signal, c'est-à-dire qu'il ne laisse passer, une fois par période, qu'une portion du signal se développant pendant $\Delta t_{\mathrm{i}}$ à partir d'un instant $t_{\mathrm{i}} \cdot t_{\mathrm{i}}=0$ pour l'impulsion de référence fournie par (9). Le retard $t_{\mathrm{i}}$ est obtenu au moyen de (10) par commande manuelle, et la durée $\Delta t_{\mathrm{i}}$ par (11). A la sortie de (4) on dispose d'un train d'impulsion de largeur $\Delta t_{\mathrm{i}}$ et de fréquence $f_{0}=1 / T_{0}$ (Fig. 7). Ce train d'impulsion est intégré dans (5) pendant un temps $n T_{0} . n$ est choisi au moyen de (12).
Le résultat de l'intégration est prélevé par un deuxième interrupteur (6) et on fait la moyenne de ces résultats dans (7). La lecture s'opère au moyen d'un voltmètre numérique (8).

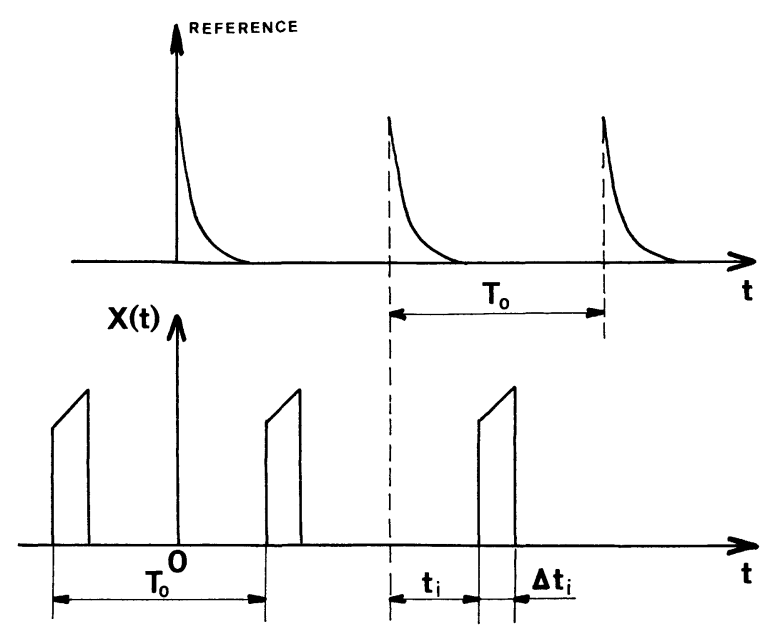

FIG. 7.

b) Analyse sommaire du fonctionnement de la chaîne de mesure. - Le filtre linéaire (3) réalise un filtrage sans déformation du signal utile. Le facteur de bruit est proportionnel à la racine carrée de la bande passante des filtres. On ne peut cependant pas avoir une bande trop étroite, à cause de l'instabilité du moteur entraînant le modulateur.

Après l'échantillonnage, le signal $x(t)$ a l'allure de la figure 7. Si $S(t)$ est le signal après intégration, on peut montrer qu'en l'absence de bruit :

$$
S\left(t_{\mathrm{i}}+n T_{0}+\Delta t_{\mathrm{i}}\right)=n \Delta t_{\mathrm{i}} \cdot x\left(t_{\mathrm{i}}+\tau\right) 0 \leqslant \tau \leqslant \Delta t_{\mathrm{i}} .
$$

La valeur de $S(t)$ prélevée par (6) est donc proportionnelle au débit du jet à l'instant $t_{\mathrm{i}}+\tau$. Si on opère une série de mesures en faisant varier $t_{\mathrm{i}}$ de 0 à $T_{0}$, on reconstitue la courbe $(\mathrm{d} n / \mathrm{d} t)_{x}$. Remarquons que $\tau$ est inconnu : il y a donc une incertitude sur le temps au maximum de $\Delta t_{\mathrm{i}}$; cependant en adoptant $\tau=\Delta t_{\mathrm{i}} / 2$, l'erreur dans la majorité des cas est beaucoup plus faible.

On peut montrer que si le bruit $\xi(t)$ qui se superpose au signal $x(t)$ a pour valeur moyenne $\mu$ et pour écart centré $\sigma$, le bruit $\varepsilon(t)$ qui se superpose au signal $S(t)$ a pour valeur moyenne $\dot{\varepsilon}$

$$
\bar{\varepsilon}=n \Delta t_{\mathrm{i}} \mu
$$

et pour écart centré :

$$
\varepsilon^{\prime}=\sqrt{\overline{(\varepsilon-\bar{\varepsilon})^{2}}}=\Delta t_{\mathrm{i}} \sigma \sqrt{n}
$$

et le rapport signal sur bruit qui était avant intégration :

$$
N_{1}=\frac{x\left(t_{\mathbf{i}}+\Delta t_{\mathbf{i}}\right)}{\sigma}
$$


devient :

$$
N_{2}=\frac{n \Delta t_{\mathrm{i}} x\left(t_{\mathrm{i}}+\Delta t_{\mathrm{i}}\right)}{\Delta t_{\mathrm{i}} \sigma \sqrt{n}}=N_{1} \sqrt{n} .
$$

L'amélioration obtenue est :

$$
\frac{N_{2}}{N_{1}}=\sqrt{n} .
$$

Le deuxième interrupteur (6) qui réalise un prélèvement tous les $n T_{0}$ se comporte comme un deuxième échantillonneur. Le moyenneur (7) est simplement un circuit $R, C$ (Fig. 8).

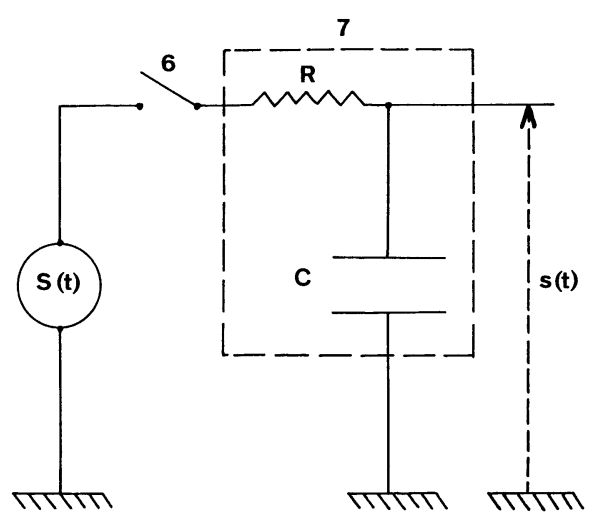

FIG. 8.

En l'absence de bruit au moment de chaque échantillonnage, $S(t)$ vaut toujours la même valeur: $S\left(t_{\mathrm{i}}+n T_{0}+\Delta t_{\mathrm{i}}\right)=S$. Et on voit facilement alors que :

$$
s(t)=\left(1-\mathrm{e}^{-n^{\prime} \theta / R C}\right) S
$$

$n^{\prime}$ étant le nombre de fermetures de (6)

$\theta$ la durée de chaque fermeture.

En présence d'un bruit à l'entrée, de valeur moyenne $\bar{\varepsilon}$ et d'écart centré $\varepsilon^{\prime}$, le bruit à la sortie a pour valeur moyenne $\bar{s}_{\mathbf{B}}$ tel que :

$$
\bar{s}_{\mathbf{B}}=\left(1-\mathrm{e}^{-n^{\prime} \theta / R C}\right) \bar{\varepsilon}
$$

et pour écart centré $S_{\mathrm{B}}$ tel que :

$$
\begin{aligned}
S_{\mathrm{B}}=\sqrt{\overline{\left(s_{\mathrm{B}}-\overline{\left.s_{\mathrm{B}}\right)^{2}}\right.}}= \\
\quad=\sqrt{\varepsilon^{\prime 2} \frac{\left(1-\mathrm{e}^{-\theta / R C}\right)\left(-\mathrm{e}^{-2 n^{\prime} \theta / R C}+1\right)}{1+\mathrm{e}^{-\theta / R C}} .}
\end{aligned}
$$

Si $n^{\prime} \theta / R C$ est suffisamment grand,

$$
\mathrm{e}^{-2 n^{\prime} \theta / R C} \simeq 0
$$

et :

$$
S_{\mathrm{B}}^{2}=\varepsilon^{\prime 2} \text { th } \frac{\theta}{2 R C} .
$$

Et le rapport signal sur bruit à la sortie s'écrit :

$$
N_{3}=\frac{S}{\varepsilon^{\prime} \sqrt{\operatorname{th} \theta / 2 R C}}=N_{2} \sqrt{\operatorname{coth} \frac{\theta}{2 R C}} .
$$

On voit donc que l'ensemble ainsi réalisé constitue un filtre non linéaire dont l'amélioration globale du rapport signal sur bruit est :

$$
\frac{N_{3}}{N_{1}}=\sqrt{n \operatorname{coth} \frac{\theta}{2 R C}} .
$$

c) Valeurs numériques adoptées. - La fréquence du premier échantillonnage a été fixée à $f_{0}=75 \mathrm{~Hz}$.

Le préamplificateur a une bande passante de $2000 \mathrm{~Hz}$ et un gain de 1000 .

Il y a 4 amplificateurs sélectifs réglés respectivement sur les fréquences $75 \mathrm{~Hz}, 225 \mathrm{~Hz}, 375 \mathrm{~Hz}, 525 \mathrm{~Hz}$.

La bande passante de l'amplificateur à $75 \mathrm{~Hz}$ est $0,75 \mathrm{~Hz}$.

La durée de la première intégration est de $4,480 \mathrm{~s}$.

La séquence d'impulsions intégrées est donc de :

$$
n=4,48 \times 75=336 \text { impulsions. }
$$

La durée du deuxième échantillonnage est $20 \mathrm{~ms}$, la constante de temps RC étant de 0,1 s.

Ainsi, entre le rapport signal sur bruit $N_{0}$ à la sortie du préamplificateur et celui $N_{3}$ à la sortie de l'appareil, l'amélioration obtenue est :

$$
\frac{N_{3}}{N_{0}}=\sqrt{\frac{2000}{0,75} \times \frac{336}{\operatorname{th} \frac{20 \times 10^{-3}}{2 \times 0,1}}}=2860 .
$$

Cependant cette amélioration du rapport signal/ bruit est obtenue aux dépens de la durée des mesures : en effet, la durée d'une mesure doit être au minimum de $1 \mathrm{mn}$, mais les signaux ainsi mesurés sont inférieurs à $0,1 \mu \mathrm{V}$.

d) Précision. - La stabilité de l'ensemble des circuits électroniques nous garantit une précision meilleure que $1 \mu$ sur le temps et $1 \%$ sur l'amplitude.

La durée minimale d'échantillonnage $\Delta t_{\mathrm{i}}$ a été fixée à $50 \mu$ s. Cependant, sur une courbe $(\mathrm{d} n(t) / \mathrm{d} t)_{x}$, on peut admettre avec une excellente approximation que l'évolution de $(\mathrm{d} n(t) / \mathrm{d} t)_{x}$ pendant une durée de $50 \mu$ s est linéaire. L'erreur sur le temps est donc de $1 \mu \mathrm{s}$. Un calcul d'erreur sur les résultats indiqués ci-dessous dans la dernière partie indique que dans ces conditions la précision de la mesure des vitesses est meilleure que $5 \%$ pour une large plage de vitesse.

IV. Résultats obtenus. - Nous avons étudié un jet d'argon pour une pression amont $P_{0}=3 \mathrm{~kg} / \mathrm{cm}^{2}$, et relevé deux courbes de débit $(\mathrm{d} n / \mathrm{d} t)_{x}$ respectivement pour $x_{1}=20 \mathrm{~cm}$ et $x_{2}=75 \mathrm{~cm}$.

La figure 9 représente la tension $v(t)$ obtenue. Le passage de $v(t)$ à $(\mathrm{d} n / \mathrm{d} t)_{x}$ a été indiqué dans la $1^{\text {re }}$ partie. 
Nous avons vu que ces courbes ont pour équation :

$$
v(t)=K_{x} \int_{0}^{t} S^{\prime}\left(t_{1}\right) f\left(t-t_{\mathrm{i}}\right) \mathrm{d} t_{\mathrm{i}} .
$$

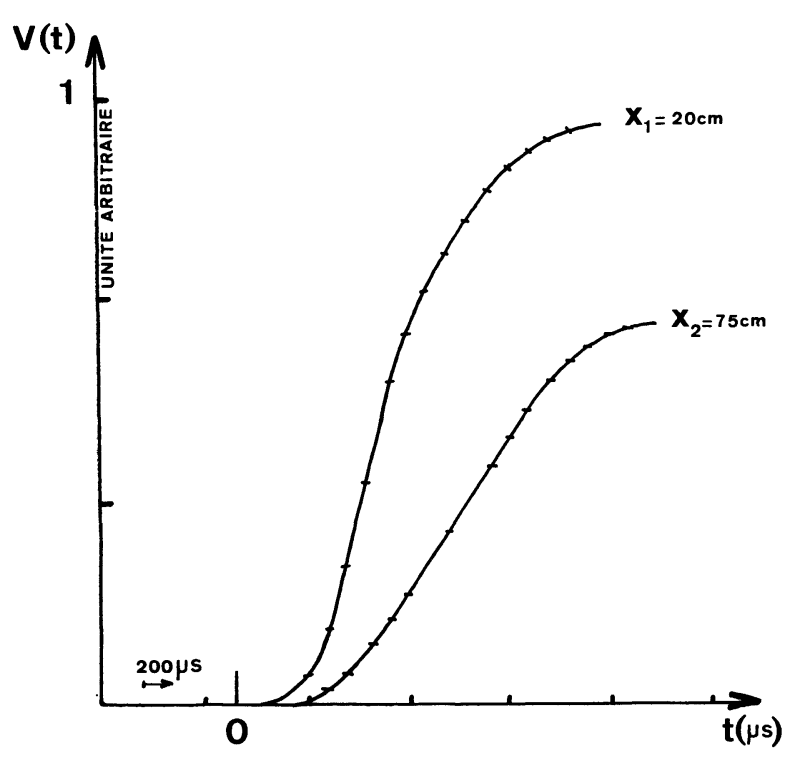

Fig. 9.

Le jet étudié étant obtenu par une détente isentropique, l'expression de sa fonction de distribution en vitesse peut être obtenue en admettant que les molécules du jet sont animées d'une vitesse d'ensemble $U$ et que la distribution des vitesses des molécules est maxwellienne autour de cette vitesse d'ensemble. Ce que nous écrivons [8], [9] :

$$
\begin{aligned}
\mathrm{d}\left(\frac{\mathrm{d} n}{\mathrm{~d} t}\right)_{\text {moléc/cm } 2 / \mathrm{s}}=n^{*} \pi^{-3 / 2}\left(\frac{v}{\alpha}\right)^{3} \mathrm{~d} \Omega \times \\
\times \exp \left[-\left(\frac{v-U}{\alpha}\right)^{2}\right]
\end{aligned}
$$

$n^{*}$ étant le nombre de molécules par unité de volume

$$
\alpha=\sqrt{\frac{2 K T}{m}}
$$

avec: $K$, constante de Boltzman; $T$, température absolue de la partie du jet en équilibre ;

$m$, la masse de la molécule d'argon;

$\mathrm{d} \Omega$, l'angle solide correspondant à l'ouverture du jet.

$\alpha$ est la vitesse la plus probable des molécules repérée par rapport à un trièdre se déplaçant à la vitesse $\mathbf{U}$.

Pour une distance $x$ donnée, la fonction $f(t)$ cherchée s'écrit dont :

$$
f(t)=K\left(\frac{x}{\alpha t}\right)^{3} \exp \left[-\left(\frac{x / t-U}{\alpha}\right)^{2}\right]
$$

$K$ étant une constante de normalisation.
D'autre part, pour des raisons de géométrie, la fonction $S^{\prime}(t)$ s'écrit :

$$
\begin{aligned}
& S^{\prime}(t)=S_{0}\left(1-\mathrm{e}^{-t / \tau}\right) \\
& {\left[r^{2} \operatorname{Arcsin} \frac{\sqrt{R \omega(2 r-R \omega)}}{r}-(r-R \omega) \sqrt{R \omega(2 r-R \omega)}\right]}
\end{aligned}
$$

où $S_{0}$ est une constante

$\tau$ la constante de temps de la chaîne électronique, soit $\tau=1500 \mu \mathrm{s}$.

$r$ le rayon du jet $=1 \mathrm{~mm}$

$R$ le rayon du disque modulateur $=45 \mathrm{~mm}$

$\omega$ la vitesse de rotation du disque en $\mathrm{rd} / \mathrm{s}=75 \pi$.

Cette fonction est obtenue en calculant la portion de section du jet non masquée par le disque modulateur.

En portant dans (4) les expressions (6) et (7) et en identifiant point à point avec les courbes expérimentales au moyen du calcul automatique, nous obtenons :

$$
\begin{aligned}
U & =399 \mathrm{~m} / \mathrm{s}, \text { à } 3 \% \text { près } \\
\alpha & =184 \mathrm{~m} / \mathrm{s} \text {, à } 12 \% \text { près } \\
V_{0} & =500 \mathrm{~m} / \mathrm{s} \text {, à } 1 \% \text { près }
\end{aligned}
$$

$V_{0}$ étant la vitesse la plus probable.

Le calcul est réalisé en cherchant le couple $(\alpha, U)$ qui minimise l'écart quadratique entre la fonction expérimentale et l'expression (4).

Nous avons trouvé pour la courbe (1) et la courbe (2) le même couple $(\alpha, U)$ optimal, l'écart quadratique étant de $4 \times 10^{-5}$ pour la courbe (1) et de $6 \times 10^{-4}$ pour la courbe (2).

La connaissance de $\alpha=184 \mathrm{~m} / \mathrm{s}$ nous permet de calculer

$$
T=\frac{m \alpha^{2}}{2 K}=82 \mathrm{oK} .
$$

Cette valeur de $T$ traduit l'efficacité de la détente : par la détente il y a orientation de l'énergie, plus $T$ est faible et plus la détente est parfaite et le rendement cinétique du jet important. Dans notre cas, nous voyons que la détente approche le maximum possible. En effet, on ne peut avoir une détente plus importante sans risque de formation de conglomérats [4].

V. Conclusion. - Nous avons cherché à obtenir la fonction de distribution en vitesse d'un jet moléculaire en relevant non pas directement la densité de probabilité, mais en travaillant sur la fonction de répartition.

En opérant des relevés au moyen d'un appareillage très élaboré constitué par un échantillonneur intégrateur à double cadence, nous avons réussi à obtenir une excellente immunité au bruit. Nous avons ainsi pu obtenir la fonction de distribution de vitesse pour un jet à faible vitesse $(V<2000 \mathrm{~m} / \mathrm{s})$ avec une précision de $5 \%$.

Nous pensons pouvoir, en utilisant la même méthode, relever la fonction de distribution de vitesse après réflexion du jet sur une surface plane. 


\section{Bibliographie}

[1] Kantrowitz (A.), Grey (J.), Rev. Sci. Inst., 1951, 22, 328.

[2] Campargue (R.), Rarefied Gas Dynamics (J.-H. de Leeuw, éditeur), Academic Press New York, 1967, vol. II, p. 279.

[3] Campargue (R.), Entropie, 1967, 18, 55.

[4] Campargue (R.), Entropie, 1969, 30, 15.

[5] Amdur (I.), STarkschell (G.), Felick (A. M.), Bertrand (R. R.), Perturbation of the velocity distribution in the scattering of molecular beams. Entropie, 1969, 30, 30.

[6] Siekhaus (W. J.), Jones (R. H.), Orlander (D. R.), Molecular beam source fabricated form multichannel arrays V, Measurement of the speed distribution. J. Appl. Phys., 1970, 41, 4392.
[7] Orlander (D. R.), Jones (R. H.), Siekhaus (W. J.), Speed distribution in the centerline beam. J. Appl. Phys., 1970, 41, 4388.

[8] Constans (A.), Daury (G.), Lostis (P.), Etude théorique de l'indicatrice de réflexion moléculaire sur une surface. C. R. Acad. Sci. Paris, 1968, 266, 695.

[9] DAURY (G.), Contribution à l'étude de la réflexion moléculaire. Annales de Physique, 1969, 4.

[10] Blanc-Lapierre (A.) et Fortet (R.), Théorie des fonctions aléatoires, Masson, 1953.

[11] Grivet (P.), BlaQuière (A.), Le bruit de fond, Masson, 1958

[12] Ragazzini (J.), Franklin (G.), Sampled-Data control systems, Mac Graw-Hill, New York, 1958, 331. 